\title{
Non- and Semi-Invasive Methods of Monitoring Eosinophilic Esophagitis
}

\author{
Calies Menard-Katcher ${ }^{\mathrm{a}-\mathrm{c}, \mathrm{e}}$ Glenn T. Furuta ${ }^{\mathrm{a}-\mathrm{e}}$ \\ ${ }^{a}$ Digestive Health Institute, Section of Pediatric Gastroenterology, Hepatology and Nutrition, Children's Hospital \\ Colorado, ${ }^{b}$ Gastrointestinal Eosinophilic Diseases Program, ${ }^{c}$ Department of Pediatrics, ${ }^{d}$ Mucosal Inflammation \\ Program, and ' University of Colorado School of Medicine, Aurora, Colo., USA
}

\section{Key Words}

Eosinophilic esophagitis - Esophageal String Test . EndoFLIP

\begin{abstract}
Background/Aims: Monitoring inflammation associated with eosinophilic esophagitis (EoE) relies on the identification of biomarkers that provide an objective measure of disease activity. To date, this metric has been the number of eosinophils in the squamous epithelial tissue. The search for alternative biomarkers as well as alternative methods to capture them has been the topic of much research. Methods: Based on clinical experience and a review of the literature, the aim of this chapter is to identify potential EoE biomarkers and methods to assess them. Results: With respect to the biomarkers, a number of candidates have arisen, including peripheral blood eosinophils, eosinophil granule proteins, Th2-related cytokines and exhaled nitric oxide. Methods to assess these biomarkers have included peripheral blood, luminal lavages and breath collections. Conclusions: Future research will identify the best clinical outcome measure for EoE. While mucosal eosinophilia currently serves as a well-defined metric of inflammation, newer research studies will continue to address whether this number correlates reliably with other patient-reported outcomes, endoscopic findings, molecular analyses or other yet to be defined biomarkers.
\end{abstract}

(c) 2014 S. Karger AG, Basel

0257-2753/14/0322-0102\$39.50/0

\section{Rationale for Monitoring Mucosal Eosinophils}

Over the course of the last decade, a number of eosinophilic esophagitis (EoE) treatments have been developed that lead to symptomatic relief in the majority of children and adults [1]. In addition, the use of swallowed steroid preparations and elimination diets can induce resolution of not only eosinophilia, but also associated features of epithelial injury. While both prospective and retrospective studies have utilized a variety of patient-reported outcome tools, peak eosinophil counts have remained the histological gold standard for therapeutic trials $[2,3]$. The primary issue directing this focus is studies demonstrating that activated eosinophils participate in pathological tissue remodeling resulting in end-organ dysfunction. With respect to EoE, it is thought that this unbridled inflammation may lead to the two known complications of EoE: food impaction and esophageal stricture.

\section{Benefits and Limitations of Endoscopic and Invasive Monitoring}

The only method to monitor EoE inflammation is with mucosal pinch biopsies obtained during endoscopy. While the procedures of endoscopy and biopsy are low risk and commonly performed, they are costly, encumber time lost from work and school, require anesthesia, and in rare cases are associated with complications. In addi-

\section{KARGER}

E-Mail karger@karger.com

www.karger.com/ddi
Glenn T. Furuta

Children's Hospital Colorado

University of Colorado School of Medicine

13123 East 16th Avenue B290, Aurora, CO 80045 (USA)

E-Mail glenn.furuta@ childrenscolorado.org 
tion, this technique is limited in its ability to capture all the inflammation that may be present in the esophagus since it samples a small fraction of the total circumferential and longitudinal mucosa. This is particularly important limitation since EoE is a panesophageal disease.

Other metrics have relied on the appearance of the mucosa with respect to the amount of exudate and edema and degree of furrowing, rings and stricture formation [4]. In addition, assessments of esophageal wall compliance with EndoFLIP and contractions with high-resolution manometry have provided real-time assessment of esophageal function $[5,6]$. These represent important steps forward in the analysis of response to treatments, but are relatively invasive techniques that require specialized equipment and interpretation.

While the current standard of care dictates that counting of eosinophils is the gold standard to monitor inflammation activity, other methods that have sought to improve on this analysis include development of scoring systems based on immunohistochemical staining for eosinophil granule proteins and molecular-based panels. Together, they represent excellent metrics to further understand pathogenetic mechanisms and response to treatments, but again they require endoscopic procedures [7-9].

\section{Biomarkers and Non-/Minimally Invasive Measurement Techniques}

Based on these concerns and limitations, the search for, and validation of, biomarkers that reflect mucosal inflammation in patients with EoE that can be captured in a non-/minimally invasive fashion continues. Below is a summary of the minimally invasive methods and biomarkers used to assess EoE disease activity.

\section{Peripheral Blood Eosinophils}

A number of studies have focused on determining whether patients with EoE also demonstrated evidence of peripheral eosinophilia [10-21]. This was based on the rationale that either patients with EoE may have elevated systemic levels of eosinopoietic or Th2 cytokines, or that patients who were experiencing a flare would have elevated peripheral eosinophils that could be captured during their migration from the bone marrow to the esophageal mucosa. Comparisons of these studies are hampered by the fact that threshold levels defining peripheral eosinophilia vary from study to study, subject populations may include atopic patients or not, and documentation of medication use that may influence counts is inconsistent. Overall, the majority, but not all, of these studies show that peripheral eosinophil levels can increase during active disease, but whether this reflects mucosal inflammation, or other atopic disease activity in general, is less certain $[48,49]$. Researchers have addressed this in a number of ways. First, investigators have attempted to tease apart peripheral eosinophilia from other factors by comparing atopic features in EoE subjects with asthma to another group who only had asthma [22]. Atopic features included peripheral eosinophilia, history of concomitant allergic diseases, family history of eosinophils and gender. They determined that peripheral eosinophilia was the most highly significant feature distinguishing patients with EoE and asthma from those only with asthma. In another study, investigators used logistic regression analysis of clinical features and determined that the combination of peripheral eosinophilia, a history of food impaction and proton pump inhibitor refractory heartburn was able to distinguish EoE from gastroesophageal reflux disease [23]. Another study examined whether phenotypic analysis of eosinophil surface markers could distinguish treated from untreated disease. Elevated levels of surface CD66 intracellular phospho-STAT1, and phospho-STAT6 were able to differentiate children with active disease from treated and healthy controls [24]. Finally, a prospective study of 47 children revealed that absolute eosinophil count, eosinophilderived neurotoxin levels and eotaxin-3 levels together correlated with esophageal inflammation [25].

Eosinophil granule proteins were measured before and after anti-IL-5 antibody treatment, demonstrating significant declines in eosinophilic cationic protein and eosinophil-derived neurotoxin followed up to 13 weeks of treatment [14]. In contrast, another study studying oral viscous budesonide in adults with EoE found no correlation of serum eosinophilic cationic protein with disease activity [14]. Finally, one study determined that eosinophil-derived neurotoxin levels provided a sustained decrease following treatment in 66 children with EoE [26]. Thus, while there appears to be a correlation of peripheral eosinophils with active inflammation, the ability for this metric alone to track disease activity is limited.

\section{Cytokines}

With advanced understanding of the molecular mechanisms of EoE, a number of studies have sought to determine whether EoE-related cytokines, including IL-5, IL-13, IL15 , CCL5, GM-CSF and others, were increased in the peripheral circulation [27-29]. In this regard, it is likely that these cytokines play a role in creation of an immunomicromilieu that is conducive to the development to of mucosal eosinophilia. The question of whether the measurement of 
peripheral cytokines will be reflective of the esophageal mucosa has led to a series of studies to assess levels of these potential surrogate markers in EoE patients. These studies have either measured Th2 cytokines in plasma of EoE patients or measured these cytokines in the supernatants of peripheral leukocytes taken from these patients.

With respect to the former studies, peripheral cytokines were measured in 10 EoE adults and 8 normal controls [28]. In this series, most of the EoE subjects were not undergoing treatment, and results showed that IL-2, IL-3, IL-5 and RANTES were greater than in the controls. Therapeutic studies targeting IL-5 have revealed a lack of consistency in elevated levels during active disease and/or decreased levels following treatment [30-35]. Straumann et al. [14] measured eotaxin before and after anti-IL- 5 antibody treatment and found that levels increased in the treatment group and that there was no change in the placebo. In another study, these same investigators measured thymus and activationregulated chemokine levels before and after treatment and found a correlation of these peripheral measurements with mucosal eosinophilia [36]. Mishra and colleagues [29] determined that serum levels of IL-15 were significantly increased in the peripheral blood of actively inflamed EoE patients and that these levels decreased following diet restriction and topical steroid treatment. Other therapeutic studies targeting IL-5 have revealed a lack of consistency in either identifying elevated levels during active disease or sustained decreased levels following treatment [30-35]. Finally, cytokine levels from normal $(\mathrm{n}=12)$ and EoE subjects with active inflammation $(\mathrm{n}=13)$ were quantified using a multiplex assay containing 84 cytokines [37]. Of these, IL13, IL-4, IL-6, IL-5, CD40L, IL-12p70 and EGF levels were significantly altered in EoE compared with normal. When these results were applied to another group of subjects with active and inactive disease, no correlations were seen.

With respect to analyses of peripheral leukocyte stimulation assays, the most recent of these studies demonstrated that invariant natural killer cells stimulated with $\alpha$-galactosylceramide and milk allergens release elevated levels of IL-4 and IL-13 [38]. While this intriguing result may replicate the in vivo microenvironment, these types of studies are hampered by variability in experimental conditions, thus preventing translation of their results to clinical utility.

Taken together, these results suggest that peripheral cytokine measurements do not consistently characterize the esophageal eosinophil levels or disease activity. In addition, analysis of peripheral cytokines, like measurements of peripheral eosinophil levels, are complicated by the confounding influence of concomitant allergic diseases and identification of validated threshold levels.

\section{Exhaled Nitric Oxide}

Fractionated exhaled nitric oxide (FeNO) has been used to assess eosinophil-predominant asthma and other atopic disorders. Since EoE is characterized by eosinophilia and basic studies suggest a link to pulmonary eosinophilia, FeNO was measured in a prospective study of 11 nonasthmatic subjects with active esophagitis before and after treatment [39]. Five patients responded histologically to topical steroids; while patients experienced a decline in FeNO, this difference did not reach statistical significance. There was a significant correlation between FeNO and symptom scores in responders. Further work will be needed to fully assess the potential utility of this test in monitoring EoE disease activity.

\section{Barium Esophagram}

Initial and subsequent descriptions of EoE showed luminal narrowing, isolated strictures and Schatzki rings to be key features of this disease. Over a quarter of a century later, barium esophagram has reemerged as a potential tool to assess pediatric subjects with EoE [40-44]. Esophagrams provide a measure of motility, mucosal irregularities and stricture formation. A retrospective analysis of 112 studies performed within 30 days of the diagnosis revealed that $52 \%$ were normal, but a minority showed findings including stricture (11\%), rings (7\%) and small-caliber esophagus (5\%) [45]. One prospective study used the minimal and maximal esophageal diameter $\left(\mathrm{ED}_{\min }\right.$ and $\mathrm{ED}_{\max }$, respectively) determined by esophagram as a therapeutic outcome [46]. Eleven adult subjects were treated for 6 weeks with topical steroids and had esophagrams performed before and after treatment. Baseline analysis revealed that the $\mathrm{ED}_{\max }$ was decreased in $50 \%$ of the EoE subjects compared to the healthy volunteer subjects. There were no statistically different changes in the $\mathrm{ED}_{\min }$ or $\mathrm{ED}_{\max }$ following treatment in the 11 patients, but in those EoE subjects with an initially abnormal esophagram, significant improvement was observed. As with FeNO, additional studies will be needed to assess this as a valuable metric for following disease activity.

\section{Luminal Secretions}

As with other luminal diseases, inflammatory mediators may be released into the cavity and reflect disease activity, e.g. sampling for eosinophils in the bronchoalveoloar lavage fluid from patients with asthma to monitor for disease activity. With respect to EoE, we have assessed the luminal inflammatory contents of EoE patient's esophagus with the Enterotest ${ }^{\mathrm{TM}}$, a string-based technology and termed this the esophageal string test [47]. The Enterotest technology was developed and suc- 
cessfully implemented in the 1970s to capture duodenal parasites. It consists of a weighted gelatin capsule filled with a nylon string. When the capsule is swallowed, the trailing string is taped to the cheek while the rest of the string is deployed into the esophagus, stomach and small intestine. The delivery capsule is released from the string, thus leaving the trailing string to capture esophageal luminal contents. We studied 42 subjects with the esophageal string test and were able to demonstrate that it was able to capture eosinophil granule proteins from the esophageal lumen and that concentrations of these proteins correlated significantly with eosinophil counts and protein concentrations found in mucosal biopsies. In addition, eosinophil-derived granule protein levels correlated significantly with disease activity. These results provide evidence that this methodology may be beneficial to monitor inflammation and disease activity.

\section{When to Monitor?}

This question continues to haunt patients and clinicians alike. Clearly, assessment of mucosal inflammation is necessary to make a diagnosis of EoE. After this initial assessment, however, the rationale for continuing monitoring still lies in the identification of meaningful clinical inflammation and complications such as strictures. While malignancy has not been reported in patients with EoE, some concerns about transformation of chronic inflammation to Barrett's esophagus still exist.

In this light, it is our practice to perform a mucosal biopsy after symptoms have improved and following 8-12 weeks of medical or diet treatment. Following this second endoscopy, a number of approaches toward following disease activity have been promoted. With respect to diet therapy, some advocate mucosal biopsy after each food has been added to the diet, some perform a biopsy after several foods and some do not perform another biopsy when the patient is doing well clinically. Since symptoms do not always match esophageal inflammation, a case can be made for any of these scenarios, but the risks and benefits of each option need to be carefully discussed with the patient and family.

'Surveillance' of the esophageal mucosa in patients with EoE following establishment of histopathological remission is a subject of even more controversy. After an effective diet is determined or medical treatment defined, some perform yearly assessments of the mucosa to insure the continued success of treatment and/or determine whether complications have developed. Another approach is to not perform another endoscopy when the patient is asymptomatic and treatments are well tolerated. This approach may involve some risk of not documenting the natural history of the disease.

Finally, assessment of the mucosa may be dictated when a previously stable patient becomes symptomatic somewhat acutely. The differential diagnosis includes recurrent EoE due to encountering an allergen, viral/fungal infection, lack of adherence to treatment or concomitant reflux disease. If these are addressed, or if patients remain symptomatic, monitoring of the mucosa may be indicated.

\section{Conclusion}

Each of these biomarkers and scenarios is presented so that clinicians can consider the best approaches for their patients. In addition, as novel therapeutics continue to be developed and studied, new techniques and biomarkers will need to be considered. Future studies documenting the impact of novel biomarkers and assessment techniques that reflect tissue eosinophilia and/or disease activity will refine the overall care of EoE patients.

\section{Acknowledgements}

This work was supported by NIH grant 1K24DK100303 (to G.T.F.).

\section{Disclosure Statement}

Dr. Menard-Katcher declares that no financial or other conflict of interest exists in relation to the content of the article. Dr. Furuta is founder of EnteroTrack, receives grant funding from $\mathrm{Nu}$ tricia and serves as a consultant to Pfizer, Meritage and Knopp.

References

1 Liacouras CA, Furuta GT, Hirano I, et al: Eosinophilic esophagitis: updated consensus recommendations for children and adults. J Allergy Clin Immunol 2011;128:3-20.e6, quiz 1-2.

2 Rothenberg ME, Aceves S, Bonis PA, et al Working with the US Food and Drug Administration: progress and timelines in understanding and treating patients with eosinophilic esophagitis. J Allergy Clin Immunol 2012;130:617-619.

-3 Fiorentino R, Liu G, Pariser AR, Mulberg AE: Cross-sector sponsorship of research in eosinophilic esophagitis: a collaborative model for rational drug development in rare diseases. J Allergy Clin Immunol 2012;130:613-616.

4 Hirano I, Moy N, Heckman MG, Thomas CS Gonsalves N, Achem SR: Endoscopic assessment of the oesophageal features of eosinophilic oesophagitis: validation of a novel classification and grading system. Gut 2013;62:489-495. 
5 Lin Z, Kahrilas PJ, Xiao Y, et al: Functional luminal imaging probe topography: an improved method for characterizing esophageal distensibility in eosinophilic esophagitis. Therap Adv Gastroenterol 2013;6:97-107.

-6 Kwiatek MA, Hirano I, Kahrilas PJ, Rothe J, Luger D, Pandolfino JE: Mechanical properties of the esophagus in eosinophilic esophagitis. Gastroenterology 2011;140:82-90.

-7 Lu TX, Rothenberg ME: Diagnostic, functional, and therapeutic roles of microRNA in allergic diseases. J Allergy Clin Immunol 2013;132:3-13.

$\checkmark 8$ Lu TX, Sherrill JD, Wen T, et al: MicroRNA signature in patients with eosinophilic esophagitis, reversibility with glucocorticoids, and assessment as disease biomarkers. J Allergy Clin Immunol 2012;129:1064-1075.e9.

-9 Protheroe C, Woodruff SA, de Petris G, et al: A novel histologic scoring system to evaluate mucosal biopsies from patients with eosinophilic esophagitis. Clin Gastroenterol Hepatol 2009;7:749-755.e11.

10 Aceves SS, Newbury RO, Dohil R, Schwimmer J, Bastian JF: Distinguishing eosinophilic esophagitis in pediatric patients: clinical, endoscopic, and histologic features of an emerging disorder. J Clin Gastroenterol 2007;41: 252-256.

11 Pasha SF, DiBaise JK, Kim HJ, et al: Patient characteristics, clinical, endoscopic, and histologic findings in adult eosinophilic esophagitis: a case series and systematic review of the medical literature. Dis Esophagus 2007;20: 311-319.

12 Lucendo Villarin AJ: Eosinophilic esophagitis - clinical manifestations, diagnosis, and treatment. Rev Esp Enferm Dig 2009;101:4959.

13 Dohil R, Newbury R, Fox L, Bastian J, Aceves $S$ : Oral viscous budesonide is effective in children with eosinophilic esophagitis in a randomized, placebo-controlled trial. Gastroenterology 2010;139:418-429.

14 Straumann A, Conus S, Grzonka P, et al: Anti-interleukin-5 antibody treatment (mepolizumab) in active eosinophilic oesophagitis: a randomised, placebo-controlled, doubleblind trial. Gut 2010;59:21-30.

$\checkmark 15$ Vindigni C, Villanacci V, Marini M, et al: Eosinophilic esophagitis: an Italian experience. Rev Esp Enferm Dig 2010;102:15-19.

16 Aceves S, Hirano I, Furuta GT, Collins MH: Eosinophilic gastrointestinal diseases - clinically diverse and histopathologically confounding. Semin Immunopathol 2012;34: 715-731.

$\checkmark 17$ Alexander JA, Jung KW, Arora AS, et al: Swallowed fluticasone improves histologic but not symptomatic response of adults with eosinophilic esophagitis. Clin Gastroenterol Hepatol 2012;10:742-749.e1.

18 Dellon ES: Diagnosis and management of eosinophilic esophagitis. Clin Gastroenterol Hepatol 2012;10:1066-1078.

19 Dellon ES: Eosinophilic esophagitis: diagnostic tests and criteria. Curr Opin Gastroenterol 2012;28:382-388.
20 Kinoshita Y, Furuta K, Ishimaura N, et al: Clinical characteristics of Japanese patients with eosinophilic esophagitis and eosinophilic gastroenteritis. J Gastroenterol 2013;48:333-339.

21 Esposito S, Marinello D, Paracchini R, Guidali P, Oderda G: Long-term follow-up of symptoms and peripheral eosinophil counts in seven children with eosinophilic esophagitis. J Pediatr Gastroenterol Nutr 2004;38:452-456.

-22 Harer KN, Enders FT, Lim KG, Alexander JA, Katzka DA: An allergic phenotype and the use of steroid inhalers predict eosinophilic oesophagitis in patients with asthma. Aliment Pharmacol Ther 2013;37:107-113.

23 von Arnim U, Wex T, Rohl FW, et al: Identification of clinical and laboratory markers for predicting eosinophilic esophagitis in adults. Digestion 2011;84:323-327.

24 Nguyen T, Gernez Y, Fuentebella J, et al: Immunophenotyping of peripheral eosinophils demonstrates activation in eosinophilic esophagitis. J Pediatr Gastroenterol Nutr 2011;53:40-47.

25 Konikoff MR, Blanchard C, Kirby C, et al: Potential of blood eosinophils, eosinophil-derived neurotoxin, and eotaxin-3 as biomarkers of eosinophilic esophagitis. Clin Gastroenterol Hepatol 2006;4:1328-1336.

26 Subbarao G, Rosenman MB, Ohnuki L, et al: Exploring potential noninvasive biomarkers in eosinophilic esophagitis in children. J Pediatr Gastroenterol Nutr 2011;53:651-658.

27 Blanchard C, Mingler MK, Vicario M, et al: IL-13 involvement in eosinophilic esophagitis: transcriptome analysis and reversibility with glucocorticoids. J Allergy Clin Immunol 2007; 120:1292-1300.

28 Johnsson M, Bove M, Bergquist H, et al: Distinctive blood eosinophilic phenotypes and cytokine patterns in eosinophilic esophagitis, inflammatory bowel disease and airway allergy. J Innate Immun 2011;3:594-604.

29 Zhu X, Wang M, Mavi P, et al: Interleukin-15 expression is increased in human eosinophilic esophagitis and mediates pathogenesis in mice. Gastroenterology 2010;139:182-193.e7.

30 Stein ML, Collins MH, Villanueva JM, et al: Anti-IL-5 (mepolizumab) therapy for eosinophilic esophagitis. J Allergy Clin Immunol 2006;118:1312-1319.

31 Conus S, Straumann A, Simon HU: Anti-IL-5 (mepolizumab) therapy does not alter IL-5 receptor alpha levels in patients with eosinophilic esophagitis. J Allergy Clin Immunol 2009; 123:269, author reply 269-270.

32 Walsh GM: Reslizumab, a humanized antiIL-5 mAb for the treatment of eosinophil-mediated inflammatory conditions. Curr Opin Mol Ther 2009;11:329-336.

33 Assa'ad AH, Gupta SK, Collins MH, et al: An antibody against IL-5 reduces numbers of esophageal intraepithelial eosinophils in children with eosinophilic esophagitis. Gastroenterology 2011;141:1593-1604.

- 34 Spergel JM, Rothenberg ME, Collins MH, et al: Reslizumab in children and adolescents with eosinophilic esophagitis: results of a double-blind, randomized, placebo-controlled trial. J Allergy Clin Immunol 2012;129: 456-463, 463.e1-3.
35 Otani IM, Anilkumar AA, Newbury RO, et al: Anti-IL-5 therapy reduces mast cell and IL-9 cell numbers in pediatric patients with eosinophilic esophagitis. J Allergy Clin Immunol 2013;131:1576-1582.

36 Straumann A, Conus S, Degen L, et al: Budesonide is effective in adolescent and adult patients with active eosinophilic esophagitis. Gastroenterology 2010;139:15261537, 1537.e1.

37 Blanchard C, Stucke EM, Rodriguez-Jimenez B, et al: A striking local esophageal cytokine expression profile in eosinophilic esophagitis. J Allergy Clin Immunol 2011;127:208-217, 217.e1-7.

38 Jyonouchi S, Smith CL, Saretta F, et al: Invariant natural killer $\mathrm{T}$ cells in children with eosinophilic esophagitis. Clin Exp Allergy 2013, E-pub ahead of print.

39 Leung J, Nguyen-Traxler A, Lee EM, et al: Assessment of fractionated exhaled nitric oxide as a biomarker for the treatment of eosinophilic esophagitis. Allergy Asthma Proc 2012; 33:519-524.

40 Feczko P, Halpert R, Zonca M: Radiographic abnormalities in eosinophilic esophagitis. Gastrointest Radiol 1985;10:321-324.

-41 Vitellas KM, Bennett WF, Bova JG, Johnson JC, Greenson JK, Caldwell JH: Radiographic manifestations of eosinophilic gastroenteritis. Abdom Imaging 1995;20:406-413.

42 Vasilopoulos S, Shaker R: Defiant dysphagia: small-caliber esophagus and refractory benign esophageal strictures. Curr Gastroenterol Rep 2001;3:225-230.

43 Vasilopoulos S, Murphy P, Auerbach A, et al: The small-caliber esophagus: an unappreciated cause of dysphagia for solids in patients with eosinophilic esophagitis. Gastrointest Endosc 2002;55:99-106.

44 White SB, Levine MS, Rubesin SE, Spencer GS, Katzka DA, Laufer I: The small-caliber esophagus: radiographic sign of idiopathic eosinophilic esophagitis. Radiology 2010;256:127-134.

45 Diniz LO, Putnum PE, Towbin AJ: Fluoroscopic findings in pediatric eosinophilic esophagitis. Pediatr Radiol 2012;42:721727.

46 Lee J, Huprich J, Kujath C, et al: Esophageal diameter is decreased in some patients with eosinophilic esophagitis and might increase with topical corticosteroid therapy. Clin Gastroenterol Hepatol 2012;10:481-486.

47 Furuta GT, Kagalwalla AF, Lee JJ, et al: The oesophageal string test: a novel, minimally invasive method measures mucosal inflammation in eosinophilic oesophagitis. Gut 2013; 62:1395-1405.

48 Boldorini R, Mercalli F, Oderda G: Eosinophilic oesophagitis in children: responders and non-responders to swallowed fluticasone. J Clin Pathol 2013;66:399-402.

49 Straumann A, Conus S, Degen L, Felder S, Kummer M, Engel H, Bussmann C, Beglinger C, Schoepfer A, Simon HU: Budesonide is effective in adolescent and adult patients with active eosinophilic esophagitis. Gastroenterology 2010;139:1526-1537, 1537.e1. 